Nuclear Science and Technology Division

\title{
ANALYSIS OF NEUTRON AND PHOTON DETECTION POSITION FOR THE CALIBRATION OF PLASTIC (BC-420) AND LIQUID (BC-501) SCINTILLATORS
}

Sara A. Pozzi, James A. Mullens, ${ }^{*}$ and John T. Mihalczo

June 2003

Prepared by

OAK RIDGE NATIONAL LABORATORY

Oak Ridge, Tennessee 27831-6283

managed by

UT-Battelle, LLC

for the

U.S. DEPARTMENT OF ENERGY

under contract DE-AC-05-00OR22725

\footnotetext{
* Engineering Science and Technology Division, ORNL
} 



\section{CONTENTS}

Page

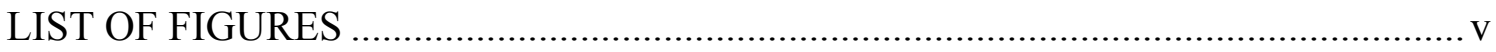

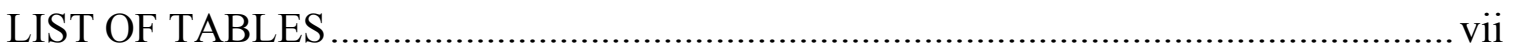

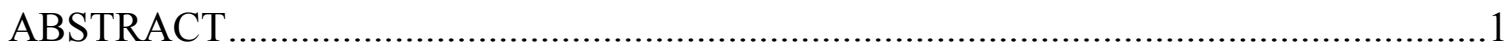

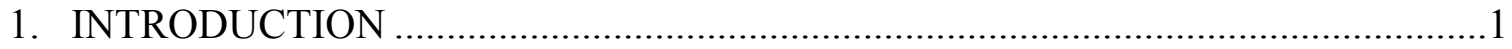

2. DETERMINATION OF THE POSITION OF DETECTION IN PLASTIC AND

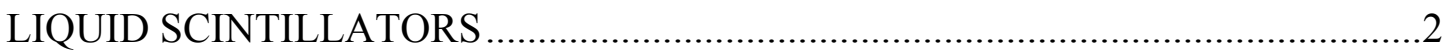

2.1 NEUTRON AND PHOTON DEPTH OF DETECTION …............................4

2.2 MULTIPLE COLLISIONS IN NEUTRON AND PHOTON DETECTION.......9

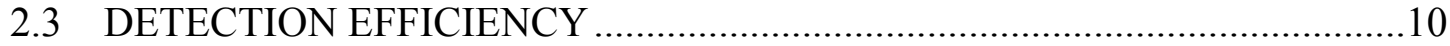

3. MEASUREMENTS FOR CALIBRATION OF BC-501 AND BC-420

SCINTILLATORS

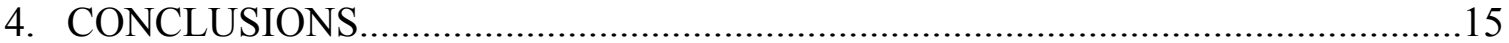

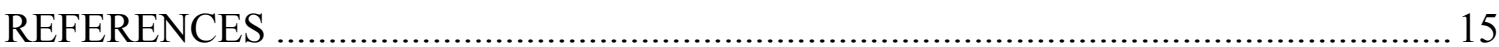





\section{LIST OF FIGURES}

Figure Page

1 Sketch of Monte Carlo simulation geometry, where $x$ is the depth of detection in in the scintillator .3

2 Monte Carlo simulation of the depth of detection in plastic scintillator for

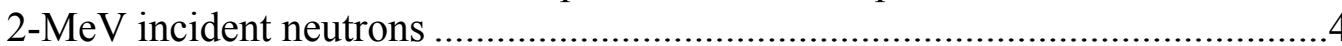

3 Average depth of neutron and photon detection in plastic scintillator, as a

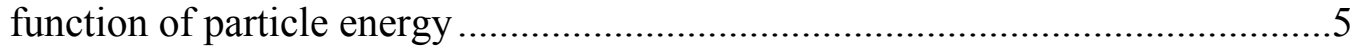

4 Average depth of neutron and photon detection in liquid scintillator, as a

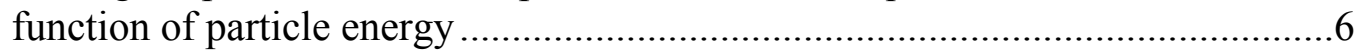

5 Monte Carlo simulation of the depth of detection in plastic scintillator for

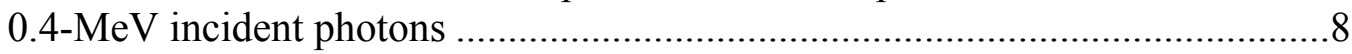

6 Number of collisions of photons of energy $0.4 \mathrm{MeV}$ occurring within the

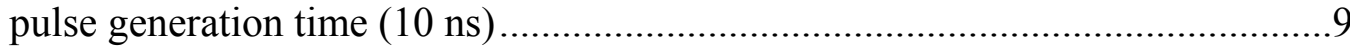

7 Number of collisions of neutrons of energy 1.0 MeV occurring within the

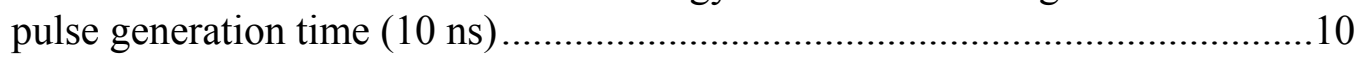

8 Monte Carlo neutron and photon detection efficiency for liquid scintillator .....11

9 Monte Carlo neutron and photon detection efficiency for plastic scintillator ....11

10 Measured neutron detection efficiency for liquid scintillator and varying CFD

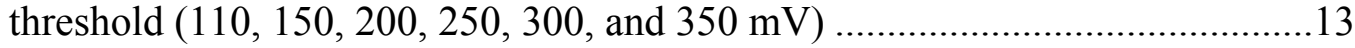

11 Light output (MeVee) of BC-420 plastic scintillator as a function of recoil particle energy $(\mathrm{MeV})$ for electrons and protons ............................................14

12 Light output (MeVee) of BC-501 liquid scintillator as a function of recoil particle energy $(\mathrm{MeV})$ for electrons and protons 



\section{LIST OF TABLES}

Table Page

1 General characteristics of Bicron scintillators ...........................................

2 Excerpt from MCNP-PoliMi output file ..........................................................2

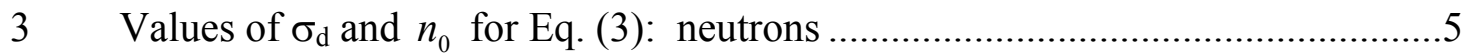

4 Average depth of detection for neutrons in scintillator ...............................

$5 \quad$ Average depth of detection for photons in scintillator....................................

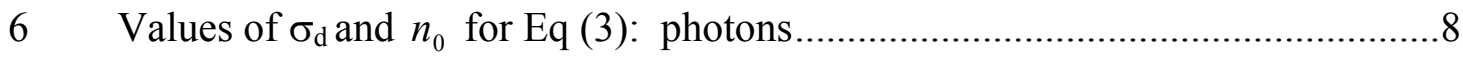

7 Neutron detection efficiency for BC-420 and BC-501 scintillators ..................12

8 Photon detection efficiency for BC-420 and BC-501 scintillators ...................12 



\title{
Analysis of Neutron and Photon Detection Position for the Calibration of Plastic (BC-420) and Liquid (BC-501) Scintillators
}

\begin{abstract}
This report presents the results of the neutron and photon calibration of a Bicron BC-420 plastic scintillator and a BC-501 liquid scintillator using a set of reference gamma sources and a Cf-252 source. The position of neutron and photon detection inside the scintillators as a function of energy is investigated by Monte Carlo simulation.
\end{abstract}

\section{INTRODUCTION}

This paper presents the calibration results for the Bicron plastic scintillator BC-420 and liquid scintillator BC-501. The calibration was performed using an instrumented Cf- 252 source and the Nuclear Materials Identification System [1]. Table 1 reports the physical characteristics of these two scintillators. The light output curves are presented for incident neutrons and photons for energies 0 to $6.5 \mathrm{MeV}$, approximately. A quadratic fit to the neutron light output as a function of energy is also given. The results are compared with those for previous experiments reported in the literature.

The proposed light output curves can be of use in simulating the detection process in these scintillators. In particular, they can be used in the post-processing of the MCNP-PoliMi code [2].

Table 1. General characteristics of Bicron scintillators

\begin{tabular}{lll}
\hline & BC-420 & BC-501 \\
\hline Density $\left(\mathrm{g} / \mathrm{cm}^{3}\right)$ & 1.032 & 0.874 \\
Atomic ratio $(\mathrm{H} / \mathrm{C})$ & 1.100 & 1.212 \\
\hline
\end{tabular}

In the past [3], a linear fit was used to relate the scintillation light output to the energy deposition by photons and neutrons in the scintillator. In the case of photons the relationship was

$$
\text { light_output }=0.99 \cdot E_{e}+0.008 \quad\left(E_{e}>0.0081 \mathrm{MeV}\right),
$$

whereas for neutrons

$$
\text { light_output }=0.23 \cdot E_{p}-0.0685 \quad\left(0.30<E_{p}<3 \mathrm{MeV}\right),
$$

where $E_{e}$ and $E_{p}$ are the energies of the recoil electron and proton, respectively, in $\mathrm{MeV}$. The light output is expressed in terms of $\mathrm{MeVee}(\mathrm{MeV}$ electron equivalent). In the case of neutrons, the linear relationship given by Eq. (2) is reasonable for $0.3<\mathrm{E}_{\mathrm{p}}<3 \mathrm{MeV}$, approximately. The calibration presented in Sect. 3 extends this energy interval to approximately $6 \mathrm{MeV}$. 


\section{DETERMINATION OF THE POSITION OF DETECTION IN PLASTIC AND LIQUID SCINTILLATORS}

The MCNP-PoliMi code output reports the neutron and gamma ray interactions with the detector at a microscopic level. An excerpt of the output file is shown in Table 2. The energy deposited by neutrons and photons in each interaction is recorded, together with the target nucleus, interaction type, and collision position.

A specifically designed post-processing code converts energy depositions into light outputs and generates a pulse by summing the light outputs recorded within the pulse generation time. The $(\mathrm{x}, \mathrm{y}, \mathrm{z})$ coordinates of the position of the interaction that generated the pulse are used to calculate the distance traveled by the neutron inside the detector.

The detector modeled is a cube of side $10.16 \mathrm{~cm}$. The detection threshold is set to $0.16 \mathrm{MeVee}$. The incident neutrons and photons come from a source placed at a distance of $100 \mathrm{~cm}$ from the front face of the detector. It follows that the angle of incidence of the particles from the source is almost perpendicular to the detector face. (The angle is $87.1^{\circ}$ to $90^{\circ}$.)

Table 2. Excerpt from MCNP-PoliMi output file

\begin{tabular}{|c|c|c|c|c|c|c|c|c|c|c|}
\hline $\begin{array}{l}\text { History } \\
\text { number }\end{array}$ & $\begin{array}{l}\text { Particle } \\
\text { number }\end{array}$ & $\begin{array}{c}\text { Projectile } \\
\text { type }^{a}\end{array}$ & $\begin{array}{l}\text { Interaction } \\
\text { type }^{b}\end{array}$ & $\begin{array}{c}\text { Target } \\
\text { nucleus }\end{array}$ & $\begin{array}{c}\text { Cell } \\
\text { number } \\
\text { of } \\
\text { collision } \\
\text { event }\end{array}$ & $\begin{array}{c}\text { Energy } \\
\text { deposited } \\
\text { in } \\
\text { collision } \\
(\mathrm{MeV}) \\
\end{array}$ & $\begin{array}{c}\text { Time } \\
\text { (shakes) }^{d}\end{array}$ & \multicolumn{3}{|c|}{$\begin{array}{c}\text { Collision position } \\
(\mathrm{x}, \mathrm{y}, \mathrm{z})\end{array}$} \\
\hline \multicolumn{11}{|l|}{$\ldots$} \\
\hline 109 & 1 & 1 & 0 & 6000 & 5 & 8.398 & 1.798 & 43.98 & -7.52 & 6.31 \\
\hline 132 & 1 & 1 & -99 & 1001 & 5 & 13.931 & 1.778 & 42.06 & -2.98 & 1.42 \\
\hline 132 & 1 & 1 & -99 & 1001 & 5 & 0.1036 & 2.075 & 42.55 & -2.34 & 2.88 \\
\hline 132 & 1 & 1 & -99 & 1001 & 5 & 0.0281 & 2.264 & 42.25 & -2.39 & 3.44 \\
\hline 132 & 1 & 1 & -99 & 1001 & 5 & 0.0131 & 2.372 & 42.16 & -2.22 & 3.63 \\
\hline
\end{tabular}

Figure 1 shows a sketch of the Monte Carlo geometry. The depth of detection, labeled $x$ in the figure, is defined as the perpendicular distance from the front face of the detector to the position of the first interaction that contributes to a pulse above the threshold. 


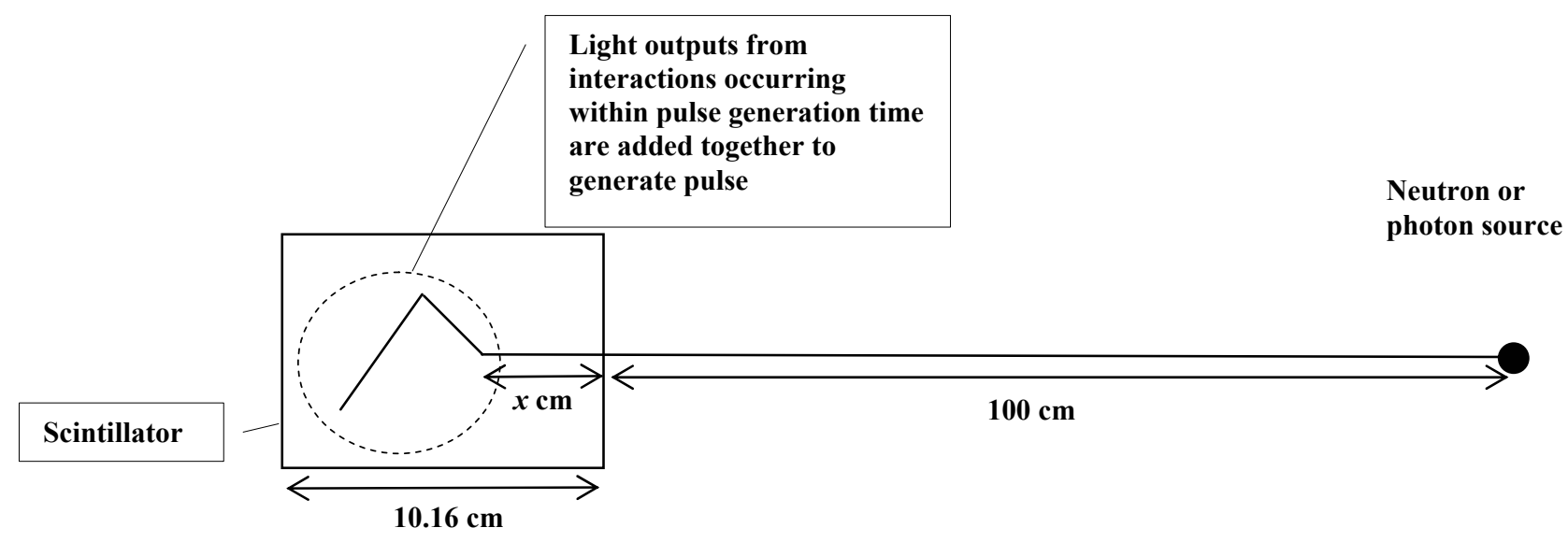

Fig. 1. Sketch of Monte Carlo simulation geometry, where $\boldsymbol{x}$ is the depth of detection in the scintillator. (Not to scale.)

\subsection{NEUTRON AND PHOTON DEPTH OF DETECTION}

Figure 2 shows the number of detections occurring inside a plastic scintillator for $2-\mathrm{MeV}$ incident neutrons as a function of the depth of detection. The data were fit to an exponential:

$$
n(x)=n_{0} \exp \left(-\sigma_{d} x\right)
$$

where $n(x)$ is the number of neutrons detected at depth $x$ inside the detector and $\sigma_{d}$ and $n_{0}$ are coefficients that depend on the incident neutron energy. The variable $\sigma_{d}$ can be interpreted as an average detection macroscopic cross section. The values of $\sigma_{d}$ and $n_{0}$ are given in Table 3 for a few neutron energies. The fit for 2-MeV neutrons is shown in Fig. 2. 


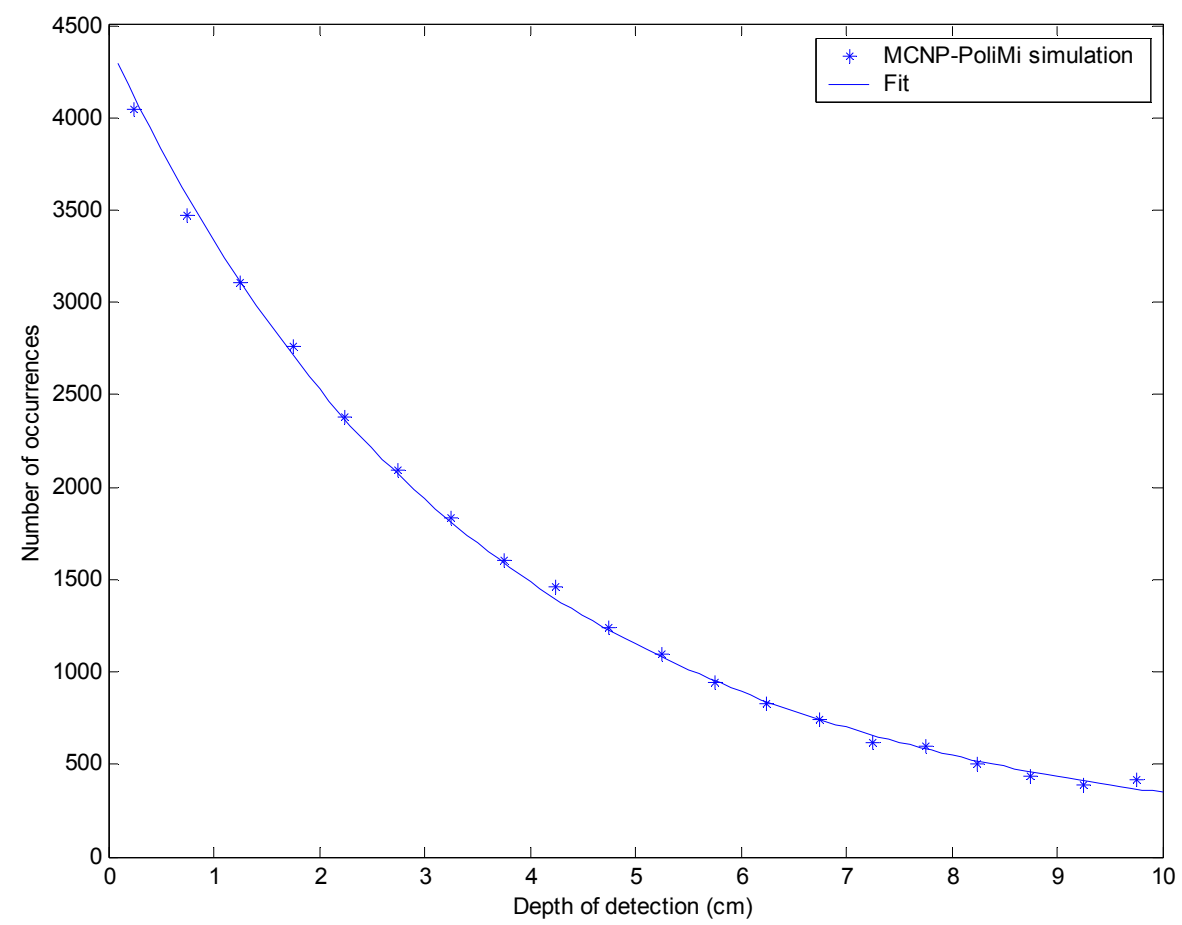

Fig. 2. Monte Carlo simulation of the depth of detection in plastic scintillator for 2-MeV incident neutrons. This simulation is indicated by stars, while the fit given by Eq. (3) is shown with the solid line.

Table 3. Values of $\sigma_{d}$ and $n_{0}$ for Eq. (3): neutrons

\begin{tabular}{lcc}
\hline $\begin{array}{l}\text { Neutron energy } \\
(\mathrm{MeV})\end{array}$ & $\sigma_{d}$ & $n_{0}$ \\
\hline 2 & 0.2544 & 4205.3 \\
3 & 0.2085 & 5480.2 \\
4 & 0.2161 & 5399.7 \\
\hline
\end{tabular}

Figure 3 shows the average depth of detection for neutrons and photons in a plastic scintillator. Neutrons of energy 1 through $7.5 \mathrm{MeV}$ and photons of energy 0.2 to $1.5 \mathrm{MeV}$ were simulated. The effect of the detection threshold is visible for photon energies lower than $0.4 \mathrm{MeV}$ : multiple interactions are necessary for the photons to deposit energy above the detection threshold. The neutron cross section of carbon accounts for the irregular character of the average depth of detection for neutrons. 
On average, the depth of detection of photons is greater than that of neutrons. The average depth of detection of $2-\mathrm{MeV}$ neutrons (most probable energy in a typical fission spectrum) is approximately $3.1 \mathrm{~cm}$. The average depth of detection for $600 \mathrm{keV}$ photons is approximately $4.05 \mathrm{~cm}$.

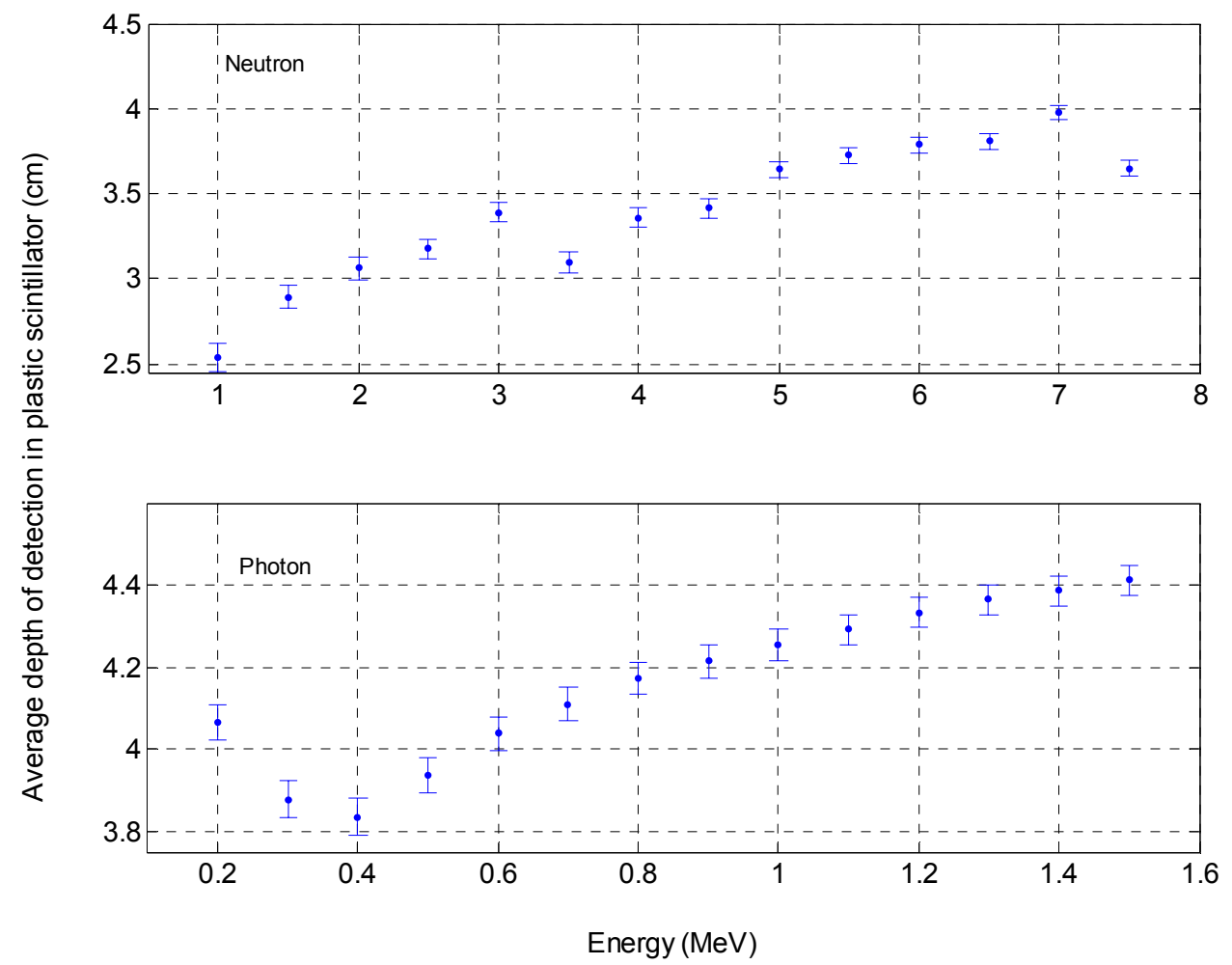

Fig. 3. Average depth of neutron and photon detection in plastic scintillator as a function of particle energy. Error bars are shown for $\pm 1 \sigma$.

Figure 4 shows the average depth of detection for neutrons and photons in a liquid scintillator. Because of the lower density of the liquid scintillator compared with the solid scintillator, the average depth of detection is slightly greater for both neutrons and photons in the liquid scintillator than in the solid. The data given in Figs. 3 and 4 are reported in Tables 4 and 5. 


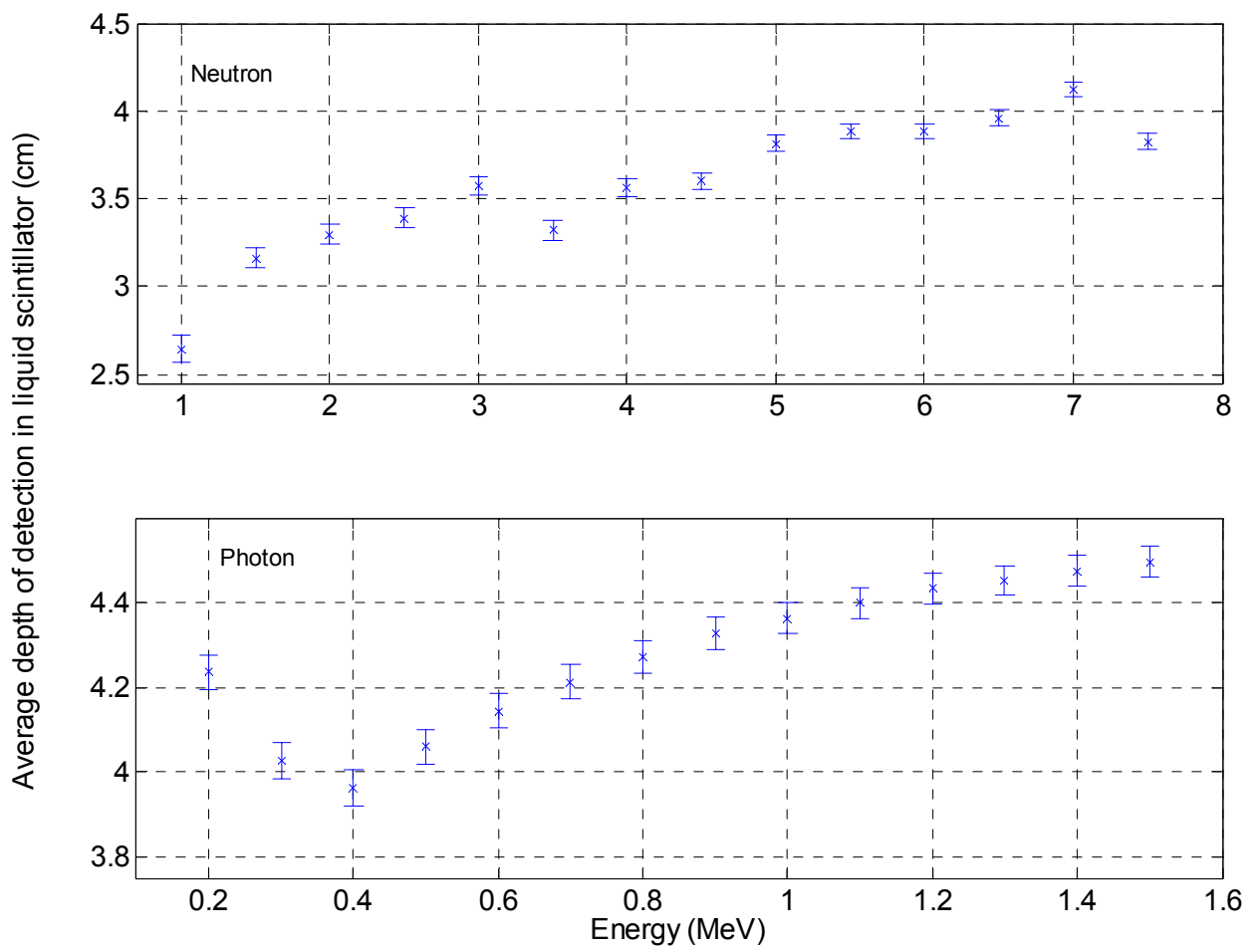

Fig. 4. Average depth of neutron and photon detection in liquid scintillator as a function of particle energy. Error bars are shown for $\pm 1 \sigma$. 
Table 4. Average depth of detection for neutrons in scintillator

\begin{tabular}{lcc}
\hline & \multicolumn{2}{c}{$\begin{array}{c}\text { Average depth of detection in scintillator }(\mathrm{cm}) \\
\text { Statistical error }( \pm 1 \sigma) \text { is shown in parenthesis }\end{array}$} \\
\hline Incident neutron energy $(\mathrm{MeV})$ & \multicolumn{1}{c}{ BC-420 } & BC-501 \\
\hline 1.0 & $2.539(0.068)$ & $2.642(0.078)$ \\
1.5 & $2.895(0.080)$ & $3.162(0.059)$ \\
2.0 & $3.062(0.064)$ & $3.298(0.057)$ \\
2.5 & $3.175(0.061)$ & $3.391(0.055)$ \\
3.0 & $3.391(0.055)$ & $3.576(0.050)$ \\
3.5 & $3.097(0.064)$ & $3.321(0.057)$ \\
4.0 & $3.358(0.056)$ & $3.561(0.051)$ \\
4.5 & $3.413(0.054)$ & $3.601(0.050)$ \\
5.0 & $3.641(0.049)$ & $3.814(0.045)$ \\
5.5 & $3.725(0.047)$ & $3.884(0.044)$ \\
6.0 & $3.788(0.046)$ & $3.884(0.043)$ \\
6.5 & $3.808(0.046)$ & $3.962(0.043)$ \\
7.0 & $3.980(0.043)$ & $4.119(0.040)$ \\
7.5 & $3.651(0.049)$ & $3.825(0.045)$ \\
\hline
\end{tabular}

Table 5. Average depth of detection for photons in scintillator

\begin{tabular}{lcc}
\hline & \multicolumn{2}{l}{$\begin{array}{l}\text { Average depth of detection in scintillator }(\mathrm{cm}) \\
\text { Statistical error }( \pm 1 \sigma) \text { is shown in parenthesis }\end{array}$} \\
\hline Incident photon energy $(\mathrm{MeV})$ & BC-420 & BC-501 \\
\hline 0.2 & $4.065(0.043)$ & $4.236(0.040)$ \\
0.3 & $3.878(0.045)$ & $4.025(0.042)$ \\
0.4 & $3.834(0.045)$ & $3.963(0.042)$ \\
0.5 & $3.938(0.043)$ & $4.060(0.041)$ \\
0.6 & $4.039(0.042)$ & $4.145(0.040)$ \\
0.7 & $4.110(0.040)$ & $4.214(0.039)$ \\
0.8 & $4.172(0.039)$ & $4.272(0.038)$ \\
0.9 & $4.214(0.039)$ & $4.327(0.037)$ \\
1.0 & $4.254(0.038)$ & $4.362(0.037)$ \\
1.1 & $4.291(0.038)$ & $4.399(0.036)$ \\
1.2 & $4.334(0.037)$ & $4.433(0.036)$ \\
1.3 & $4.364(0.037)$ & $4.452(0.036)$ \\
1.4 & $4.387(0.036)$ & $4.475(0.035)$ \\
1.5 & $4.412(0.036)$ & $4.497(0.035)$ \\
\hline
\end{tabular}

Figure 5 shows the number of detections occurring inside a plastic scintillator for 0.4-MeV incident photons as a function of the depth of detection. Approximately $50 \%$ of the photons are detected in the first $5.5 \mathrm{~cm}$ of the plastic scintillator. Table 6 reports the values of $\sigma_{d}$ and $n_{0}$ for a few photon energies. 


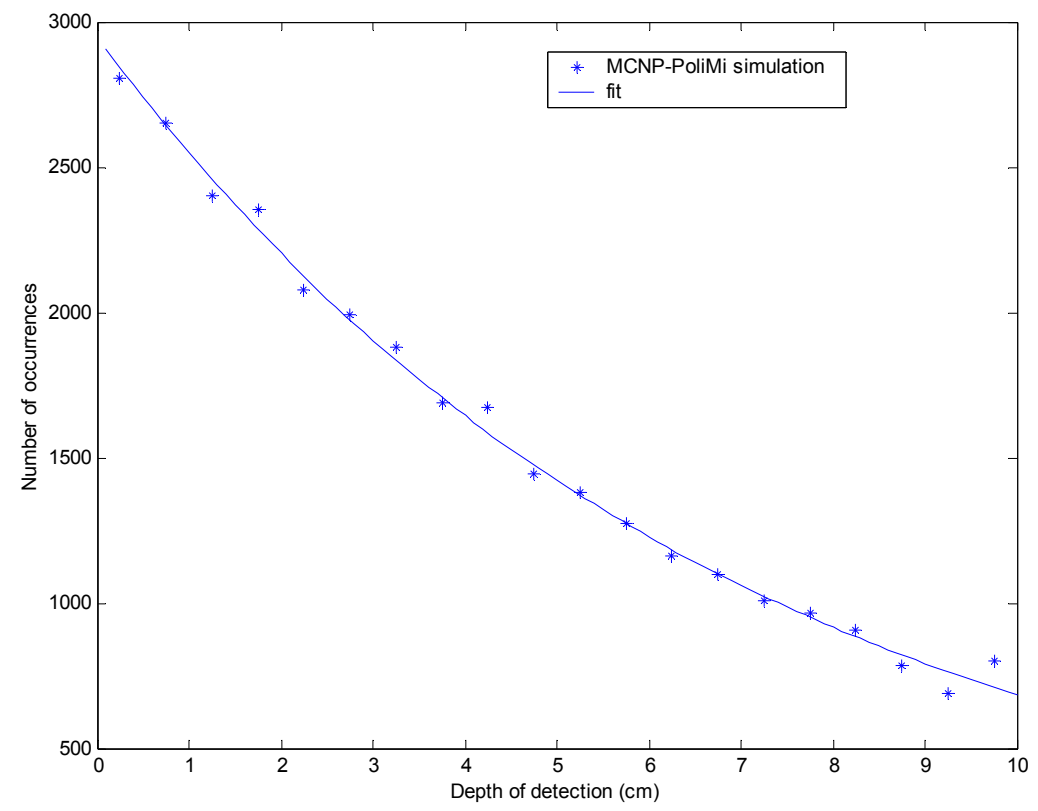

Fig. 5. Monte Carlo simulation of the depth of detection in plastic scintillator for 0.4-MeV incident photons. This simulation is indicated by stars, while the fit given by Eq. (3) is shown with the solid line.

Table 6. Values of $\sigma_{d}$ and $n_{0}$ for Eq. (3): photons

\begin{tabular}{lcc}
\hline $\begin{array}{l}\text { Photon energy } \\
(\mathrm{MeV})\end{array}$ & $\sigma_{d}$ & $n_{0}$ \\
\hline 0.4 & 0.1460 & 2950 \\
0.6 & 0.1179 & 3116 \\
0.8 & 0.1012 & 2959 \\
1.0 & 0.0910 & 2776 \\
\hline
\end{tabular}




\subsection{MULTIPLE COLLISIONS IN NEUTRON AND PHOTON DETECTION}

The detector model takes into account the possibility of multiple collisions occurring within a specified time, which is referred to as pulse generation time (PGT). The PGT for the scintillators evaluated is set to $10 \mathrm{~ns}$. This value can be justified in part by considering the order of magnitude of the pulse rise time at the anode of the scintillator photomultiplier tube.

Figure 6 shows the number of collisions occurring within the PGT for $0.4-\mathrm{MeV}$ photons incident on a plastic scintillator. These data refer to histories that result in a detection (light output exceeding the $0.16-\mathrm{MeVee}$ threshold). Approximately $57 \%$ of the detections are a result of multiple photon interactions (two or more successive interactions).

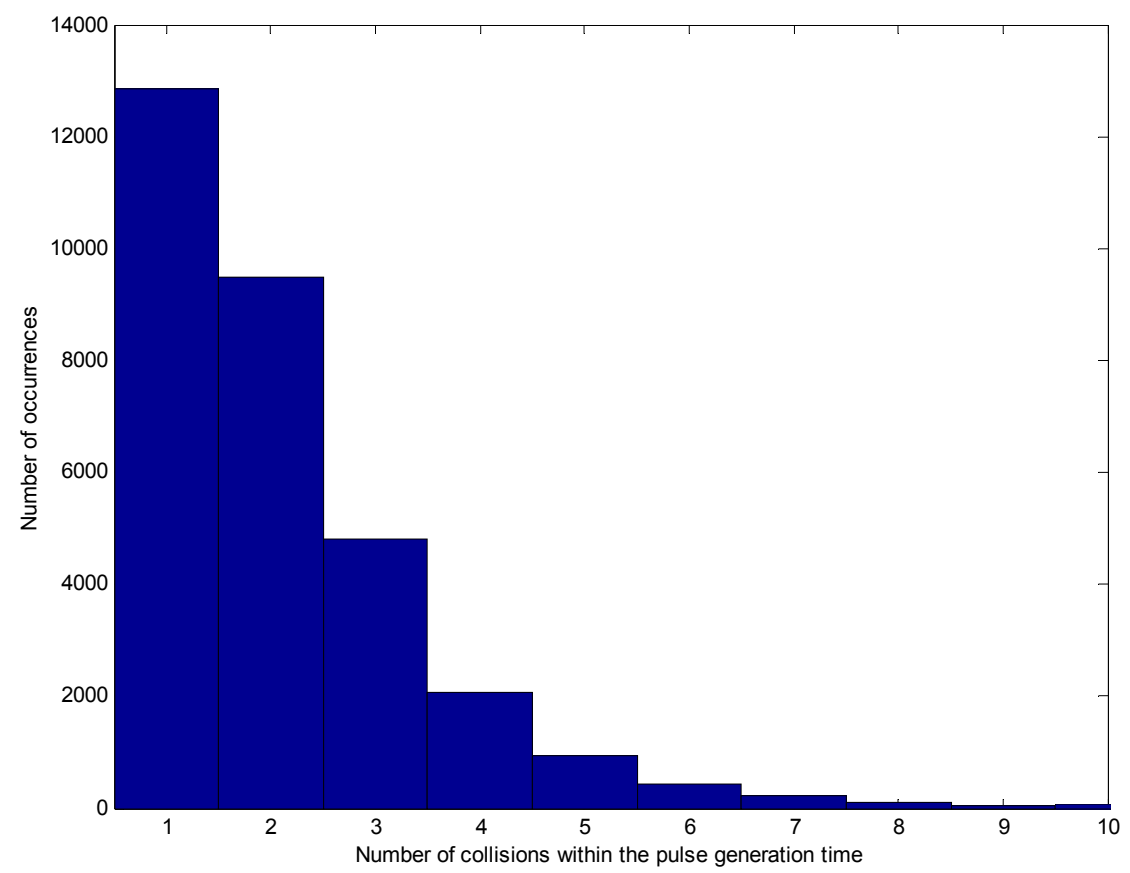

Fig. 6. Number of collisions of photons of energy $0.4 \mathrm{MeV}$ occurring within the pulse generation time (10 ns).

Figure 7 shows the number of collisions occurring within the PGT for 1-MeV neutrons incident on a plastic scintillator. In this case, approximately $44 \%$ of the detections are a result of a multiple neutron interactions (two or more successive interactions). 


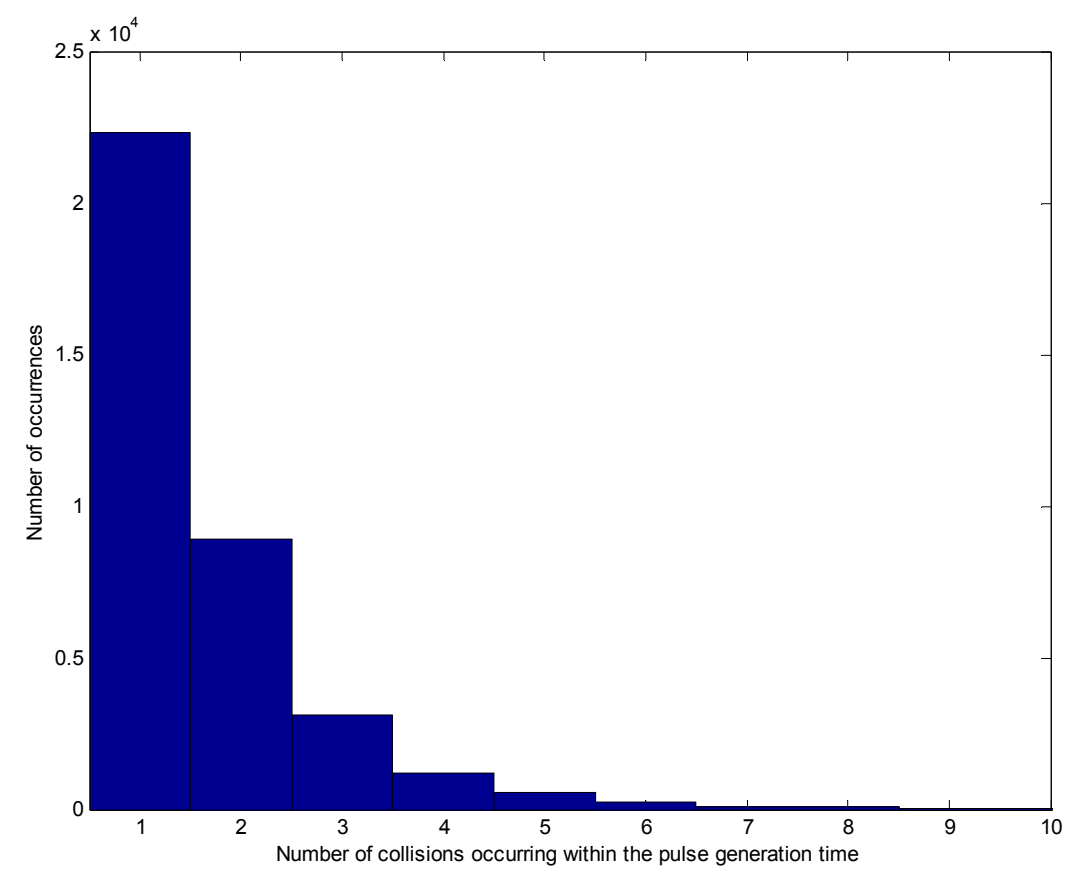

Fig. 7. Number of collisions of neutrons of energy $1.0 \mathrm{MeV}$ occurring within the pulse generation time $(10 \mathrm{~ns})$.

\subsection{DETECTION EFFICIENCY}

Figures 8 and 9 show the Monte Carlo simulation results of the detection efficiency for neutrons and photons incident on the liquid and plastic scintillators, respectively. The intrinsic detection efficiency is defined as the number of particles that are detected by the detector divided by the number of particles impinging on the detector. The simulation geometry is given in Fig. 1. The detection threshold is set to $0.16 \mathrm{MeVee}$.

As it can be seen, the maximum neutron detection efficiency is approximately $25 \%$ greater than the maximum photon detection efficiency in the range of energies considered. 


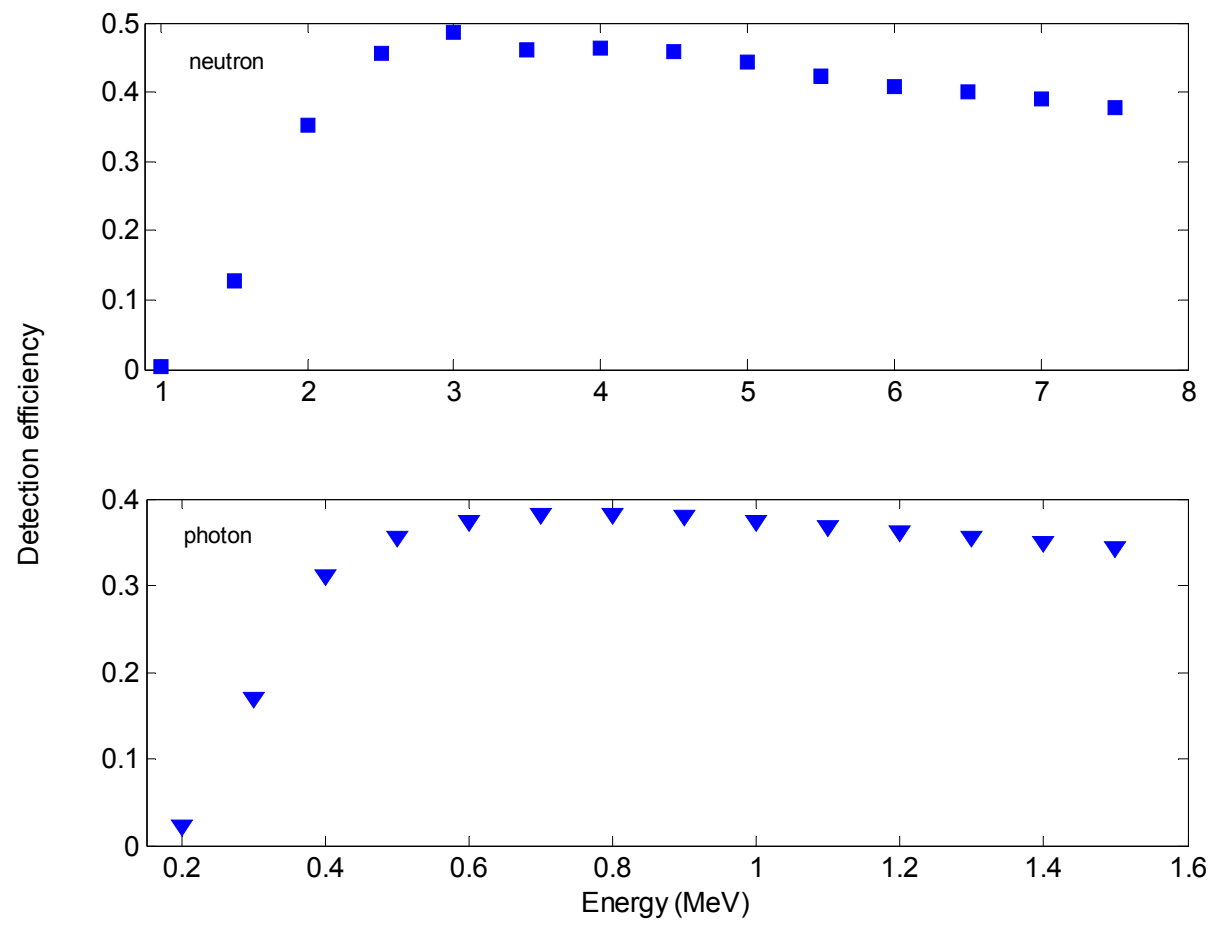

Fig. 8. Monte Carlo neutron and photon detection efficiency for liquid scintillator.

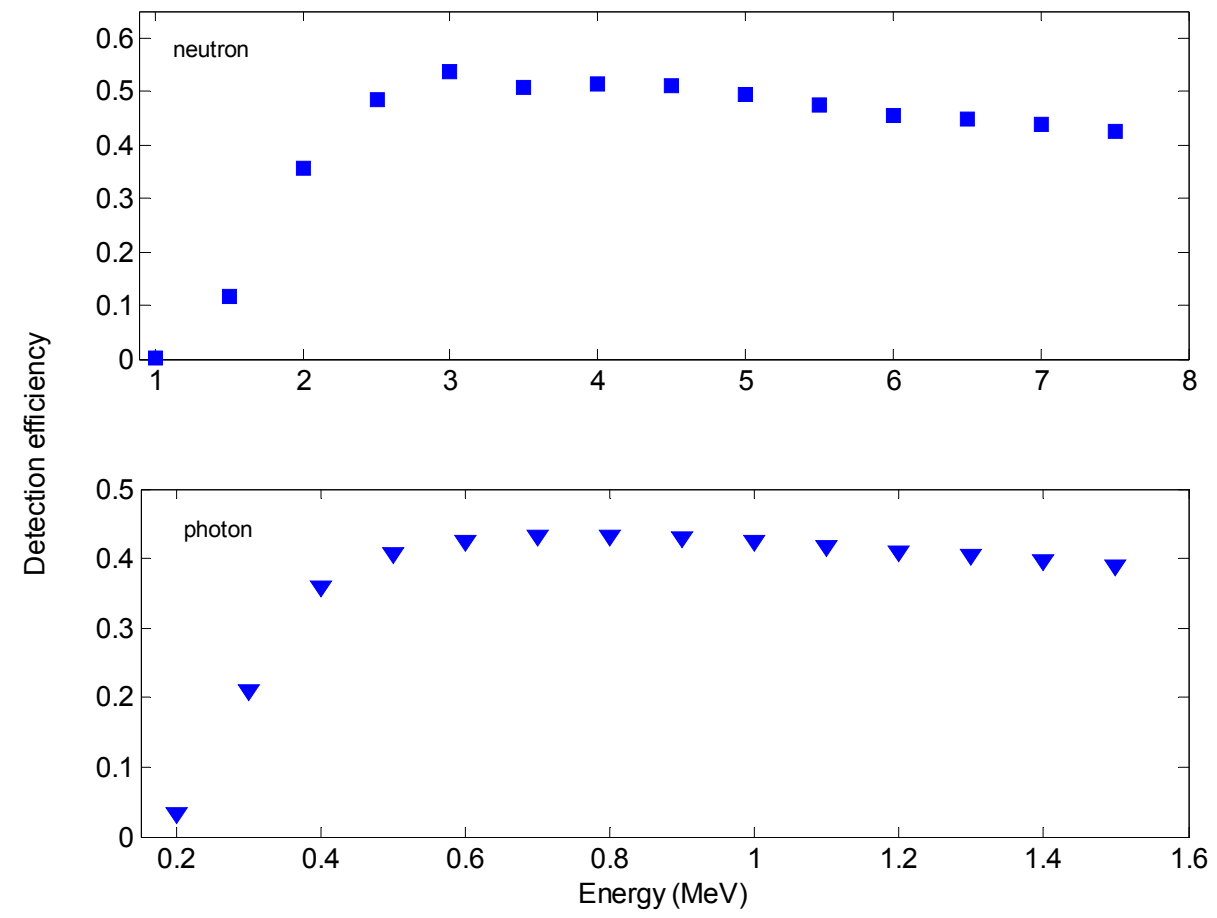

Fig. 9. Monte Carlo neutron and photon detection efficiency for plastic scintillator. 
Table 7. Neutron detection efficiency for BC-420 and BC-501 scintillators

\begin{tabular}{lcc}
\hline Incident neutron energy $(\mathrm{MeV})$ & \multicolumn{2}{c}{ Detection efficiency } \\
& \multicolumn{2}{c}{ Statistical error $( \pm 1 \sigma)$ is shown in parenthesis } \\
\cline { 2 - 3 } & BC-420 & BC-501 \\
\hline 1.0 & $0.003(0.0002)$ & $0.003(0.0002)$ \\
1.5 & $0.116(0.0012)$ & $0.128(0.0012)$ \\
2.0 & $0.356(0.0020)$ & $0.353(0.0020)$ \\
2.5 & $0.485(0.0024)$ & $0.455(0.0023)$ \\
3.0 & $0.536(0.0025)$ & $0.487(0.0024)$ \\
3.5 & $0.506(0.0024)$ & $0.462(0.0023)$ \\
4.0 & $0.513(0.0024)$ & $0.464(0.0023)$ \\
4.5 & $0.510(0.0024)$ & $0.457(0.0023)$ \\
5.0 & $0.495(0.0024)$ & $0.443(0.0023)$ \\
5.5 & $0.475(0.0024)$ & $0.423(0.0022)$ \\
6.0 & $0.455(0.0023)$ & $0.407(0.0022)$ \\
6.5 & $0.448(0.0023)$ & $0.400(0.0022)$ \\
7.0 & $0.440(0.0023)$ & $0.391(0.0021)$ \\
7.5 & $0.424(0.0022)$ & $0.377(0.0022)$ \\
\hline
\end{tabular}

Table 8. Photon detection efficiency for BC-420 and BC-501 scintillators

\begin{tabular}{lcc}
\hline Incident photon energy $(\mathrm{MeV})$ & \multicolumn{2}{c}{ Detection efficiency } \\
& Statistical error $( \pm 1 \sigma)$ is shown in parenthesis \\
\cline { 2 - 3 } & BC-420 & BC-501 \\
\hline 0.2 & $0.034(0.001)$ & $0.022(0.001)$ \\
0.3 & $0.210(0.001)$ & $0.172(0.001)$ \\
0.4 & $0.361(0.002)$ & $0.313(0.002)$ \\
0.5 & $0.407(0.002)$ & $0.356(0.002)$ \\
0.6 & $0.427(0.002)$ & $0.375(0.002)$ \\
0.7 & $0.433(0.002)$ & $0.382(0.002)$ \\
0.8 & $0.434(0.002)$ & $0.383(0.002)$ \\
0.9 & $0.430(0.002)$ & $0.381(0.002)$ \\
1.0 & $0.425(0.002)$ & $0.376(0.002)$ \\
1.1 & $0.418(0.002)$ & $0.369(0.002)$ \\
1.2 & $0.412(0.002)$ & $0.363(0.002)$ \\
1.3 & $0.404(0.002)$ & $0.356(0.002)$ \\
1.4 & $0.397(0.002)$ & $0.350(0.002)$ \\
1.5 & $0.391(0.002)$ & $0.344(0.002)$ \\
\hline
\end{tabular}




\section{MEASUREMENTS FOR CALIBRATION OF BC-501 AND BC-420 SCINTILLATORS}

Calibration measurements were performed with a $9.5 \times 9.5 \times 10.16 \mathrm{~cm} \mathrm{BC}-420$ plastic scintillator and a cylindrical cell of BC-501 liquid scintillator, having diameter $11.75 \mathrm{~cm}$ and thickness $10.2 \mathrm{~cm}$. The neutron time-of-flight data from an instrumented Cf- 252 source were used to obtain the neutron efficiency of the detectors [1]. An example of the efficiency of the plastic scintillator for varying constant fraction discriminator (CFD) thresholds is shown in Fig. 10. A linear fit to the rising edge of the efficiency curve was used to determine the neutron energy threshold corresponding to the CFD setting. These data, together with a photon calibration performed using a number of reference gamma sources, were used to find a relationship between the energy deposited by the particles in the detectors and the light output.

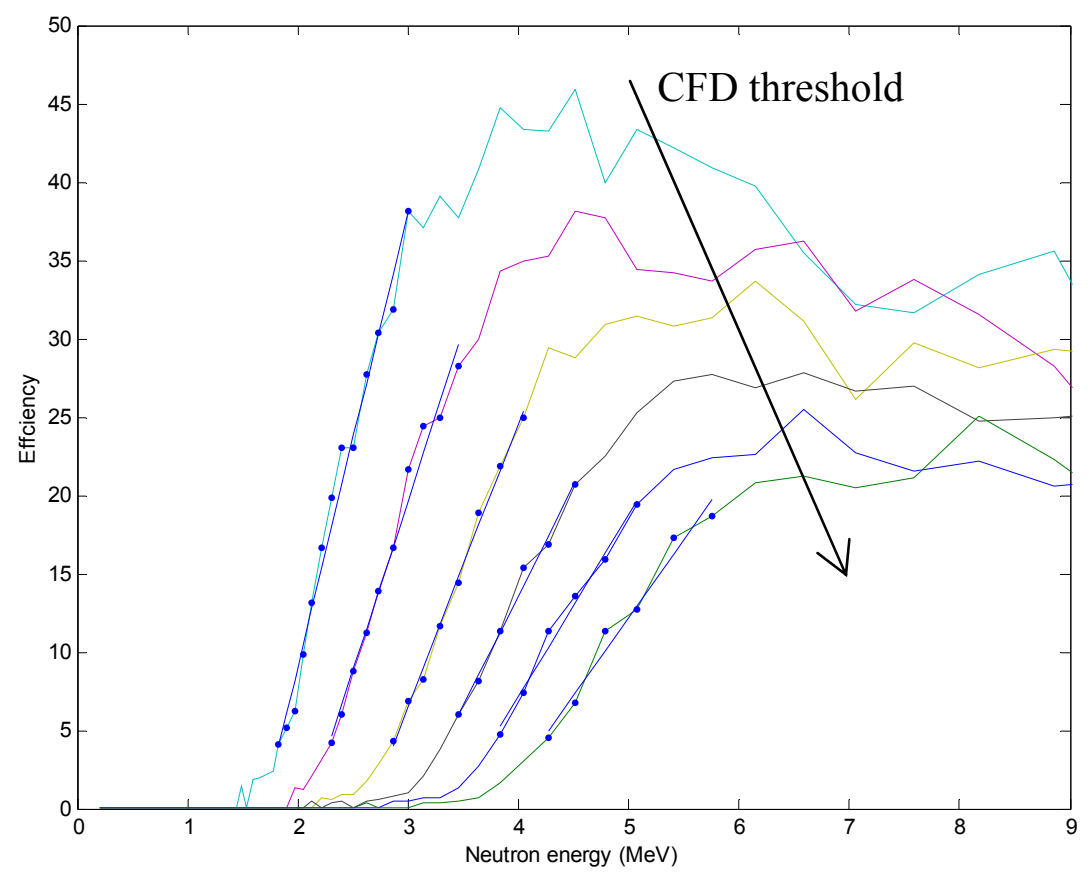

Fig. 10. Measured neutron detection efficiency for liquid scintillator and varying CFD threshold $(110,150,200,250,300$, and $350 \mathrm{mV})$.

For photons, the linear relationship given by Eq. (1) was confirmed. For neutrons, the new calibration gives

$$
\text { light_output }=0.0364 \cdot E_{n}^{2}+0.125 E_{n}
$$

for the plastic scintillator and 


$$
\text { light_output }=0.0350 \cdot E_{n}^{2}+0.1410 E_{n}
$$

for the liquid scintillator.

Plots of the experimental data and the fits given by Eqs.4. (1), (4), and (5) are shown in Figs. 11 and 12, together with the results obtained by Czirr and colleagues [4].

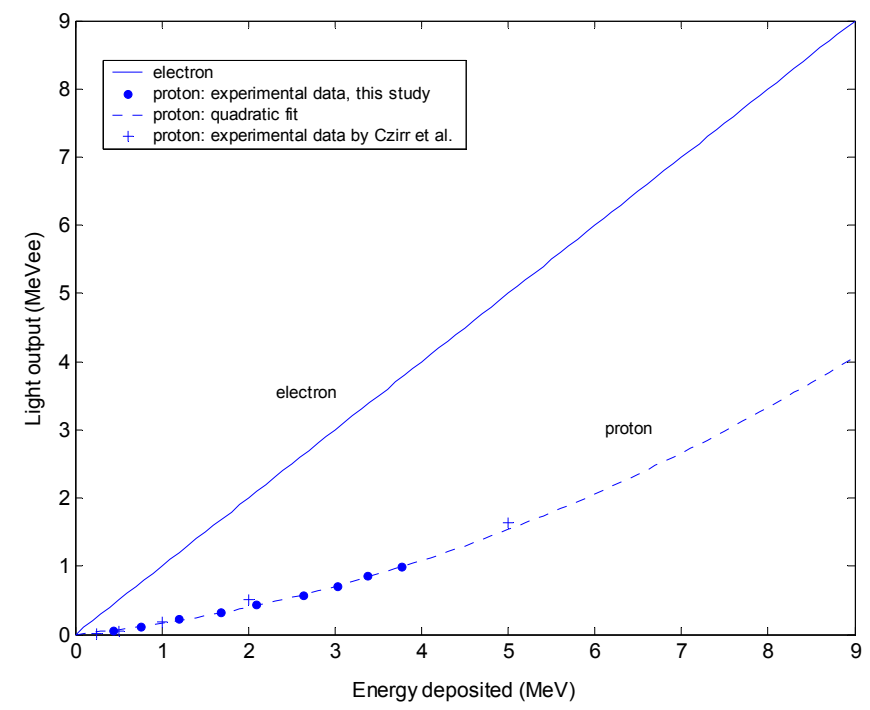

Fig. 11. Light output (MeVee) of BC-420 plastic scintillator as a function of recoil particle energy $(\mathrm{MeV})$ for electrons and protons.

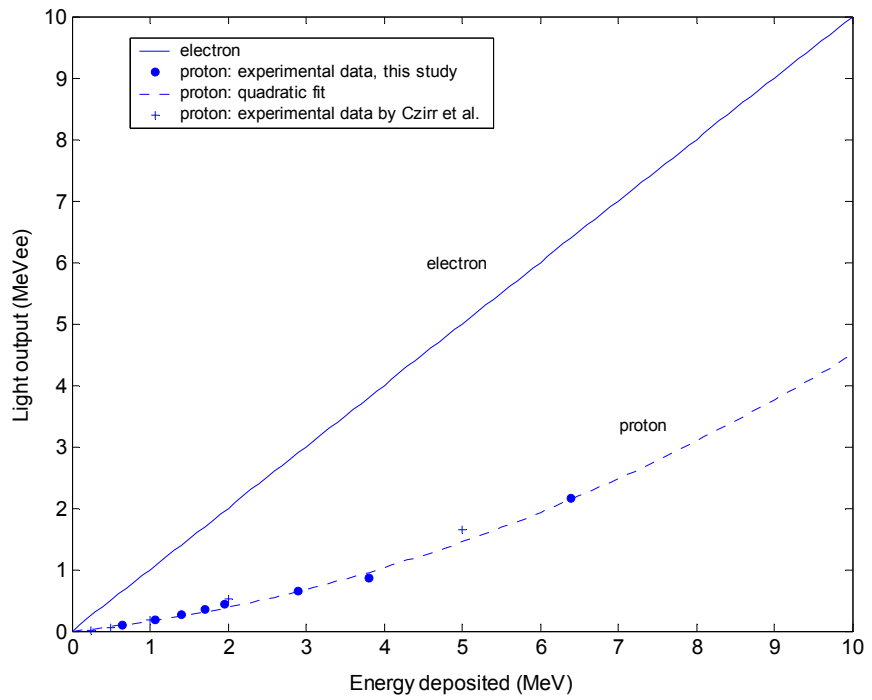

Fig. 12. Light output (MeVee) of BC-501 liquid scintillator as a function of recoil particle energy ( $\mathrm{MeV}$ ) for electrons and protons. 


\section{CONCLUSIONS}

In this work, the results of a neutron and gamma ray calibration for plastic and liquid scintillators are presented. The results are in good agreement with results found in the literature. A linear fit for photon light output and a quadratic fit for neutron light output are proposed.

The Monte Carlo code MCNP-PoliMi was used to analyze the depth of detection of neutrons and photons in plastic and liquid scintillators. This information can be used to determine the optimal thickness of scintillation detectors used in different applications. The consistency between measurements and simulations allows for a better understanding of the detection process and improved interpretation of measurements using these types of detectors.

This application of MCNP-PoliMi illustrates one of many possible ways to post-process the information present in the output file. In fact, the detection model is not limited to scintillation detector types.

\section{REFERENCES}

1. J. T. Mihalczo, J. A. Mullens, J. K. Mattingly, and T. E. Valentine, "Physical Description of Nuclear Materials Identification System (NMIS) Signatures," Nuclear Instruments and Methods in Physics Research Section A 450, 531-555, (August 11, 2000).

2. Enrico Padovani and Sara A. Pozzi, "MCNP-PoliMi ver. 1.0. User's Manual," CESNEF021122, Library of the Department of Nuclear Engineering, Polytechnic of Milan, Italy, (2002).

3. M. Marseguerra, E. Padovani, and S. A. Pozzi, "Use of the MCNP-POLIMI Code for TimeCorrelation Safeguards Measurements," Progress in Nuclear Energy, 43(1-4) 305-311, (2003).

4. J. B. Czirr, D. R. Nygren, and C. D. Zafiratos, "Calibration and Performance of a Neutron Time of Flight Detector," Nuclear Instruments and Methods 31, 226-232, (1964). 